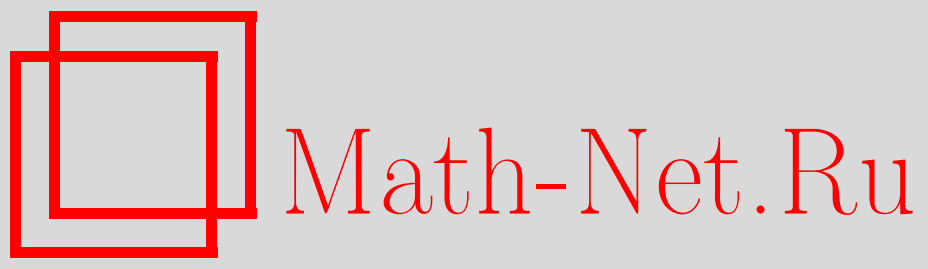

С. В. Асташкин, Обобщенное неравенство Хинчина в симметричных пространствах, Функи. анализ и его прил., 2008, том 42, выпуск 2, 78-81

DOI: https://doi.org/10.4213/faa2905

Использование Общероссийского математического портала MathNet.Ru подразумевает, что вы прочитали и согласны с пользовательским соглашением

http://www . mathnet.ru/rus/agreement

Параметры загрузки:

IP : 3.80 .181 .102

26 апреля 2023 г., 16:02:01

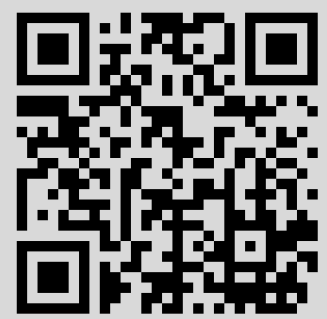




\title{
ЛитеРАтУРА
}

[1] М. Ш. Бирман, М. З. Соломяк, в кн.: Итоги науки и техники. Математический анализ, т. 14, ВИНИТИ, М., 1977, 5-58. [2] А. С. Андреев, Матем. сб., 197:2 (2006), $17-34$.

Военно-морской институт радиоэлектроники им. А. С. Попова

Поступило в редакцию 13 июня 2006 г.

УДК 517.982.27

\section{Обобщенное неравенство Хинчина в симметричных пространствах}

\author{
(C) 2008. С. В. АСТАШКИН
}

1. Введение. Пусть $r_{k}(t)=\operatorname{sign} \sin 2^{k} \pi t(k=1,2, \ldots)$ - функции Радемахера, рассматриваемые на отрезке $[0,1]$. Согласно классическому неравенству Хинчина [1], для любого $p>0$ существует константа $C_{p}>0$, такая, что для произвольных вещественных $a_{k}(k=1,2, \ldots)$ выполнено неравенство $\left\|\sum_{k=1}^{\infty} a_{k} r_{k}\right\|_{L_{p}} \leqslant C_{p}\left(\sum_{k=1}^{\infty} a_{k}^{2}\right)^{1 / 2}$.

Это соотношение вызвало большое количество исследований и обобщений, нашло многочисленные применения в различных разделах анализа [2]. В частности, в 1975 г. Родин и Семенов [3] доказали, что в случае симметричных пространств (с.п.) $X$ на $[0,1]$ неравенство

$$
\left\|\sum_{k=1}^{\infty} a_{k} r_{k}\right\|_{X} \leqslant C\left(\sum_{k=1}^{\infty} a_{k}^{2}\right)^{1 / 2}
$$

имеет место тогда и только тогда, когда $X$ содержит $G$, где $G$ - сепарабельная часть пространства Орлича $L_{N_{2}}$, построенного по функции $N_{2}(u)=e^{u^{2}}-1$. В этой работе будет дано решение аналогичной задачи в случае, когда вместо скалярных кратных функций Радемахера рассматриваются произвольные независимые функции, в среднем равные нулю. Точнее, будет дан ответ на вопрос, для каких с.п. $X$ существует $C>0$, такое, что для всех последовательностей независимых функций $\left\{f_{k}\right\}_{k=1}^{\infty} \subset X$, удовлетворяющих условиям $\int_{0}^{1} f_{k}(t) d t=0$ $(k=1,2, \ldots)$ и $\left(\sum_{k=1}^{\infty} f_{k}^{2}\right)^{1 / 2} \in X$, выполнено неравенство

$$
\left\|\sum_{k=1}^{\infty} f_{k}\right\|_{X} \leqslant C\left\|\left(\sum_{k=1}^{\infty} f_{k}^{2}\right)^{1 / 2}\right\|_{X} .
$$

Заметим, что если класс последовательностей независимых функций, в среднем равных нулю, заменить на более обширный класс мартингальных разностей, то ответ на этот вопрос известен: необходимое и достаточное условие на $X$ состоит в положительности нижнего индекса Бойда этого пространства, т. е. $\alpha_{X}>0$ [4].

В нашей ситуации центральная роль будет принадлежать так называемому свойству Круглова, введенному и интенсивно изучавшемуся Браверманом [5]. Основной результат этой заметки (теорема 1) в терминах этого свойства дает 
характеризацию сепарабельных или максимальных с.п., в которых обобщенное неравенство Хинчина (2) выполнено для произвольных последовательностей независимых функций, в среднем равных нулю. Второй результат (теорема 2) показывает, что то же свойство оказывается полезным при изучении свойств подпространства с.п., порожденного системой Радемахера с независимыми векторными коэффициентами.

2. Определения и обозначения. Банахово пространство $X$ вещественнозначных функций, измеримых по Лебегу на интервале $[0, \alpha)(0<\alpha \leqslant \infty)$, называется симметричным (или перестановочно инвариантным) пространством (с.п.), если из того, что $y \in X$ и $x^{*}(t) \leqslant y^{*}(t)(t \in[0, \alpha))$, следует, что $x \in X$ и $\|x\|_{X} \leqslant\|y\|_{X}$. Здесь $x^{*}(t)$ - невозрастающая непрерывная справа перестановка функции $|x(s)|$, т. е. $x^{*}(t)=\inf \{\tau \geqslant 0: \lambda(\{s \in[0, \alpha):|x(s)|>\tau\}) \leqslant t\}, t>0$ ( $\lambda$ - мера Лебега).

Если $X$ - с.п. на интервале $[0, \alpha)$, то двойственное (или ассоииированное) пространство $X^{\prime}$ состоит из всех измеримых функций $y$, для которых $\|y\|_{X^{\prime}}:=$ $\sup \left\{\int_{0}^{\alpha}|x(t) y(t)| d t: x \in X,\|x\|_{X} \leqslant 1\right\}<\infty$. С.п. $X$ называется максимальным (обладает свойством Фату), если естественное вложение этого пространства в его второе двойственное $X^{\prime \prime}$ - сюръективная изометрия. Это эквивалентно тому, что из условий $x_{n} \in X(n=1,2, \ldots), x_{n} \rightarrow x$ п.в. на $[0, \alpha)$ и $\sup _{n=1,2, \ldots}\left\|x_{n}\right\|_{X}<\infty$ следует, что $x \in X$. Заметим, что все важные в приложениях с.п. (пространства Орлича, Марцинкевича, Лоренца и т.д.) либо максимальны, либо сепарабельны. Важной характеристикой с.п. являются его индексы Бойда $\alpha_{X}$ и $\beta_{X}$, связанные с оператором растяжения; всегда $0 \leqslant \alpha_{X} \leqslant \beta_{X} \leqslant 1$ [6, теорема 2.4.4]. Для любого с.п. $X$ на $[0,1]$ имеют место непрерывные вложения $L_{\infty}[0,1] \subset X \subset L_{1}[0,1][6$, теорема 2.4.1].

Если $X$ - с.п. на $[0,1]$, то пространство $Z_{X}^{2}$, введенное в [7], состоит из всех $f \in\left(L_{1}+L_{\infty}\right)(0, \infty)$, таких, что

$$
\|f\|_{Z_{X}^{2}}:=\left\|f^{*} \chi_{[0,1]}\right\|_{X}+\left\|f^{*} \chi_{[1, \infty)}\right\|_{L_{2}[1, \infty)}<\infty .
$$

Нетрудно показать, что квазинорма $\|\cdot\|_{Z_{X}^{2}}$ эквивалентна некоторой норме, и поэтому $Z_{X}^{2}$ является с.п. на $[0, \infty)$. Если $\left\{f_{k}\right\}_{k=1}^{\infty}-$ последовательность функций, определенных на $[0,1]$, то через $\left\{\bar{f}_{k}\right\}_{k=1}^{\infty}$ далее будет обозначаться последовательность дизъюнктных одинаково распределенных с функциями $f_{k}$ функций на $[0, \infty)$, например, $\bar{f}_{k}(t)=f_{k}(t-k+1) \chi_{[k-1, k)}(t)$. Подробнее о с.п. см. монографии [6], [8], [9].

Пусть $f$ - измеримая функция (случайная величина) на $[0,1]$. Через $\pi(f)$ обозначим случайную величину $\sum_{i=1}^{N} f_{i}$, где $f_{i}-$ независимые копии функции $f$, а $N$ - случайная величина, имеющая распределение Пуассона с параметром 1, независимая относительно последовательности $\left\{f_{i}\right\}$. Следующее свойство было введено и интенсивно изучалось Браверманом [5], использовавшим при этом некоторые вероятностные конструкции Круглова [10].

Определение 1. С.п. $X$ на $[0,1]$ обладает свойством Круглова (иными словами, $X \in \mathbb{K})$, если из того, что $f \in X$, следует, что $\pi(f) \in X$.

Грубо говоря, с.п. $X$ принадлежат классу $\mathbb{K}$, если оно расположено достаточно «далеко» от $L_{\infty}$. В частности, это верно, если $X \supset L_{p}$ при некотором $p<\infty$ [5, теорема 1.2 , с. 16]. Кроме того, если $L_{N_{\gamma}}$ - экспоненциальное пространство 
Орлича, построенное по функции $N_{\gamma}(u)=e^{u^{\gamma}}-1$, то $L_{N_{\gamma}} \in \mathbb{K}$ тогда и только тогда, когда $0<\gamma \leqslant 1[5$, с. 42$]$.

\section{3. Формулировка результатов.}

Теорема 1. Пусть $X$ - сепарабельное или максимальное с.n. на [0, 1]. Следующие условия эквивалентны:

(a) $X \in \mathbb{K}$;

(b) существует $C>0$, такое, что для любой последовательности независимых функиий $\left\{f_{k}\right\}_{k=1}^{\infty} \subset X$, удовлетворяющ, у условиям $\int_{0}^{1} f_{k}(t) d t=0$ $(k=1,2, \ldots)$ u $\sum_{k=1}^{\infty} \bar{f}_{k} \in Z_{X}^{2}$, выполнено неравенство

$$
\left\|\sum_{k=1}^{\infty} f_{k}\right\|_{X} \leqslant C\left\|\sum_{k=1}^{\infty} \bar{f}_{k}\right\|_{Z_{X}^{2}}
$$

(с) существует $C>0$, такое, что для любой последовательности независимых функиий $\left\{f_{k}\right\}_{k=1}^{\infty} \subset X$, удовлетворяющих условиям $\int_{0}^{1} f_{k}(t) d t=0$ $(k=1,2, \ldots)$ u $\left(\sum_{k=1}^{\infty} f_{k}^{2}\right)^{1 / 2} \in X$, выполнено неравенство $(2)$.

Замечание 1. Неравенство, противоположное к (3), выполнено в любом с.п. $X$ [7]. Кроме того, в [7] доказано, что (3) верно, если $X \supset L_{p}$ при некотором $p<\infty$. Последнее условие, как уже говорилось, более ограничительно, чем условие (а). В частности, теорема 1 показывает, что соотношения (2) и (3) имеют место в экспоненциальном пространстве Орлича $L_{N_{\gamma}}$, если $0<\gamma \leqslant 1$.

Замечание 2. Более слабое, нежели положительность нижнего индекса Бойда, условие $X \in \mathbb{K}$, с одной стороны, показывает разницу между свойствами независимых функций, в среднем равных нулю, и общих мартингальных разностей [4]. С другой стороны, оно сильнее, чем условие $X \supset G$, обеспечивающее выполнение неравенства (1), частного случая неравенства (2) для скалярных кратных одной последовательности - системы Радемахера.

Пусть $X$ - с.п. на $I=[0,1]$, а $X(I \times I)$ - соответствующее с.п. на квадрате $I \times I$, т. е. $\|f\|_{X(I \times I)}=\left\|f^{*}\right\|_{X}$.

Теорема 2. Если с.п. X сепарабельно или максимально, то следующие условия эквивалентны:

( $\alpha) X \in \mathbb{K}$;

( $\beta$ ) существует $C>0$, такое, что для любой последовательности независимых функиий $\left\{f_{k}\right\}_{k=1}^{\infty} \subset X$

$$
C^{-1}\left\|\left(\sum_{k=1}^{\infty} f_{k}^{2}\right)^{1 / 2}\right\|_{X} \leqslant\left\|\sum_{k=1}^{\infty} f_{k}(s) r_{k}(t)\right\|_{X(I \times I)} \leqslant C\left\|\left(\sum_{k=1}^{\infty} f_{k}^{2}\right)^{1 / 2}\right\|_{X} ;
$$

$(\gamma)$ существует $C>0$, такое, что для любой последовательности независимьх функиий $\left\{f_{k}\right\}_{k=1}^{\infty} \subset X$

$$
C^{-1}\left\|\left(\sum_{k=1}^{\infty} f_{k}^{2}\right)^{1 / 2}\right\|_{X} \leqslant \int_{0}^{1}\left\|\sum_{k=1}^{\infty} f_{k}(s) r_{k}(t)\right\|_{X} d t \leqslant C\left\|\left(\sum_{k=1}^{\infty} f_{k}^{2}\right)^{1 / 2}\right\|_{X} .
$$


Замечание 3. Утверждение теоремы 2 интересно сопоставить с двумя известными результатами. Первый из них утверждает, что в любом сепарабельном или максимальном с.п. $X$ эквивалентны следующие условия: (i) существует $C>0$, такое, что для произвольной последовательности функций $\left\{f_{k}\right\}_{k=1}^{\infty} \subset X$ выполнено неравенство (4) и (ii) $\alpha_{X}>0$ (импликация (ii) $\Longrightarrow$ (i) доказана в [8, предложение 2.d.1], a (i) $\Longrightarrow$ (ii) - в [11]). Второй (неравенство Морэ) говорит о том, что если $X$ есть $q$-вогнутая банахова решетка [8, определение 1.d.3] при некотором $q<\infty$, то существует $C>0$, такое, что (5) выполнено для произвольной последовательности функций $\left\{f_{k}\right\}_{k=1}^{\infty} \subset X$ [8, теорема 1.d.6(i)]. Заметим, что, с одной стороны, в обоих утверждениях коэффициенты ряда произвольные функции, с другой - условия выполнения неравенств (4) и (5) в них гораздо более ограничительные, чем условие $(\alpha)$.

Замечание 4. При доказательстве результатов заметки был использован операторный подход к изучению свойства Круглова, развитый в работах [12], [13].

\title{
ЛитерАтУРА
}

[1] A. Khintchine, Math. Z., 18 (1923), 109-116. [2] Г. Пешкир, А. Н. Ширяев, УМН, 50:5 (1995), 3-62. [3] V. A. Rodin, E. M. Semenov, Anal. Math., 1:3 (1975), 207-222. [4] W. B. Johnson, G. Schechtman, Israel J. Math., 64:3 (1988), 267-275. [5] M. Sh. Braverman, Independent Random Variables and Rearrangement Invariant Spaces, Cambridge Univ. Press, Cambridge, 1994. [6] С. Г. Крейн, Ю. И. Петунин, Е. М. Семенов, Интерполяция линейных операторов, Наука, М., 1978. [7] W. B. Johnson, G. Schechtman, Ann. Probab., 17:2 (1989), 789-808. [8] J. Lindenstrauss, L. Tzafriri, Classical Banach spaces II. Function spaces, Springer-Verlag, Berlin-Heidelberg-New York, 1979. [9] C. Bennett, R. Sharpley, Interpolation of Operators, Academic Press, Boston, 1988. [10] В. М. Круглов, ТВП, 15:2 (1970), 331-336. [11] С. В. Асташкин, М. Ш. Браверман, в кн.: Операторные уравнения в функциональных пространствах, Изд-во ВГУ, Воронеж, 1986, 3-10. [12] С. В. Асташкин, Ф. А. Сукочев, Матем. заметки, 76:4 (2004), 483-489. [13] S. V. Astashkin, F. A. Sukochev, Israel J. Math., 145 (2005), 125-156.

Самарский госуниверситет

e-mail: astashkn@ssu.samara.ru
Поступило в редакцию

25 октября 2006 г.

\section{УДК 514.76}

\section{Препятствия к эквивалентности пуассоновых структур вблизи симплектического листа полупростого и компактного типа}

\author{
(c) 2008. Ю. М. Воробьев
}

В настоящей заметке мы формулируем необходимые и достаточные условия для существования изоморфизма пуассоновых структур в окрестности симплектического листа полупростого и компактного типа. В частности, показано, 